\title{
Robotic Force Stabilization for Beating Heart Intracardiac Surgery
}

\author{
Shelten G. Yuen ${ }^{1}$, Michael C. Yip ${ }^{1}$, Nikolay V. Vasilyev ${ }^{2}$, Douglas P. Perrin ${ }^{2}$, \\ Pedro J. del Nido ${ }^{2}$, and Robert D. Howe ${ }^{1,3}$ \\ 1 Harvard School of Engineering and Applied Sciences, Cambridge, MA \\ 2 Department of Cardiovascular Surgery, Children's Hospital Boston, MA \\ 3 Harvard-MIT Division of Health Sciences \& Technology, Cambridge, MA
}

\begin{abstract}
The manipulation of fast moving, delicate tissues in beating heart procedures presents a considerable challenge to surgeons. We present a new robotic force stabilization system that assists surgeons by maintaining a constant contact force with the beating heart. The system incorporates a novel, miniature uniaxial force sensor that is mounted to surgical instrumentation to measure contact forces during surgical manipulation. Using this sensor in conjunction with real-time tissue motion information derived from 3D ultrasound, we show that a force controller with feed-forward motion terms can provide safe and accurate force stabilization in an in vivo contact task against the beating mitral valve annulus. This confers a $50 \%$ reduction in force fluctuations when compared to a standard force controller and a $75 \%$ reduction in fluctuations when compared to manual attempts to maintain the same force.
\end{abstract}

\section{Introduction}

Beating heart surgical procedures remove the need for cardiopulmonary bypass, which has a number of well known and serious side effects for patients [1]. However, surgical manipulation inside the beating heart is challenging because heart motion exceeds the approximately one $\mathrm{Hz}$ human tracking bandwidth [2]. The mitral valve annulus, for instance, traverses most of its trajectory and undergoes three direction changes in approximately $100 \mathrm{~ms}$, making the application of a constant, firm force for surgical tasks like mitral valve annuloplasty difficult [3] .

These challenges have spurred recent interest in robotically-assisted interventions 3 4 56]. In prior work, we developed a 3D ultrasound-guided motion compensation system that uses an actuated, handheld robotic instrument to track beating intracardiac structures [3]. While this approach increases surgical capabilities in certain tasks [36, problems arise when the instrument comes into sustained contact with tissue because small positional errors can lead to significant forces and the deformation of tissue by the instrument reduces the usefulness of subsequent position measurements. In this work we consider a more direct approach by using force control of the instrument against the surgical target. We further show that feeding forward tissue motion information from 3D ultrasound can improve the performance of a force controller in this application. 
A

Tissue Motion Estimates
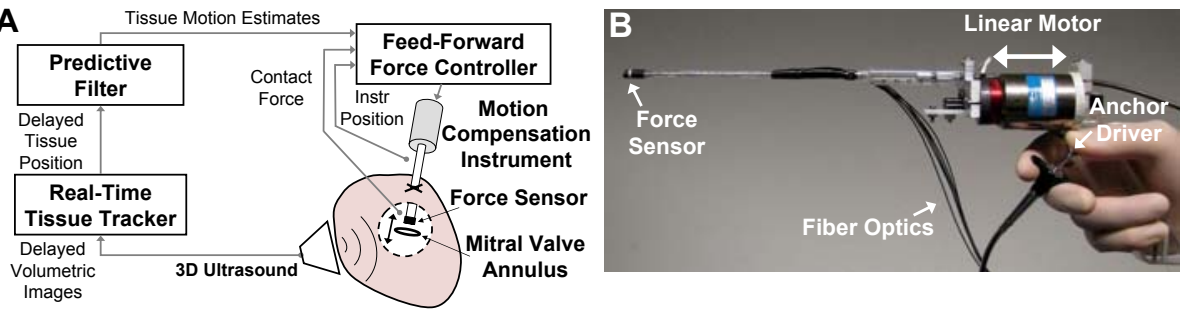

Fig. 1. (A) System overview and (B) motion compensation instrument. The instrument is controlled to a constant force against beating heart structures. Feed-forward motion estimates are derived from a 3D ultrasound tissue tracker and predictive filter.

Beating heart force control requires the development of a force sensor that can be used inside the heart. Force sensors have been developed for minimally invasive investigations [7, but they are not capable of integration with existing surgical instruments for simultaneous measurement and modification of beating heart tissue. Safety is also an important consideration when robots are used in surgery. While force control stability and performance have been studied in general settings [8], they have not been examined for beating heart applications.

In this work, we present a robotic force stabilization system for beating heart tissue manipulation (Fig. 1A). The system uses a novel, miniature uniaxial force sensor that can measure contact forces inside the heart. It is mounted to an actuated, handheld surgical instrument that we term the motion compensation instrument (Fig. 1B) for interaction with moving intracardiac structures. We describe the design of a feed-forward force controller for enhanced safety and accuracy, with feed-forward information provided by real-time 3D ultrasound tissue tracking. We validate the system in the context of beating heart mitral valve annuloplasty in an in vivo experiment in a Yorkshire pig model.

\section{Force Sensor Design}

A number of considerations guide the design of the force sensor for our system. First, the sensor should be located at the instrument tip to accurately measure contact forces. Second, its use inside the heart dictates that it be small, completely sealed from blood, and electrically passive to avoid disrupting conduction in the heart. Finally, to be useful in beating heart mitral annuloplasty, the sensor must be compatible with the deployment of surgical anchors.

Optically-based sensing is attractive in this setting because it does not require electrical trasmission to the sensor, has low noise, is readily miniaturized, and permits inexpensive, disposable sensors. The sensing principle relies on measuring small displacements of a reflective plate relative to the ends of optical fiber pairs (Fig. 2). Three pairs of optical fibers, with each pair comprised of one transmitting and one receiving fiber, are placed in an equilateral triangle formation at the base of the sensor to ensure that the reflective plate deflection 


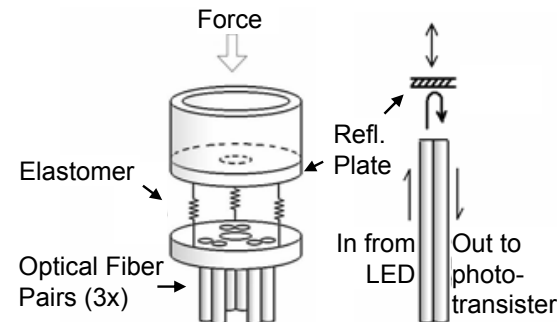

Fig. 2. Tip forces displace a reflective plate and increase light intensity to the receiving optical fibers

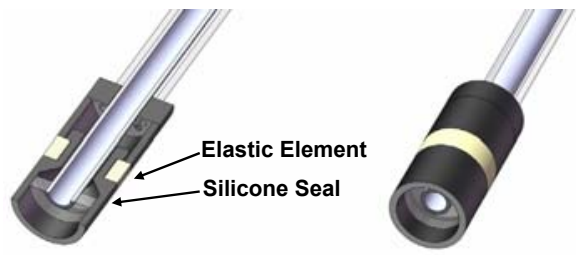

Fig. 3. A 14 gauge needle passes through the sensor to deploy surgical anchors

is captured entirely. An elastomer element is placed between the optical fiber ends and the reflective plate to convert force to displacement. The displacement modifies the light intensity measured by the receiving fiber, which is converted to a voltage by a phototransistor circuit.

Figs. $1 \mathrm{~B}$ and 3 show the prototype force sensor. It is built to encompass a 14 gauge needle for the deployment of surgical anchors. Polysiloxane elastomer provides low modulus and hysteresis. The rigid housing is made of Delrin for good appearance in ultrasound images. The external diameter and length of the force sensor are $5.5 \mathrm{~mm}$ and $12 \mathrm{~mm}$, respectively. A thin film of silicone seals the exterior surface of the sensor to shield the internal components from fluid motion. Characterization of the force sensor against a commercial sensor (ATI mini40) indicates that our sensor has a $0.17 \mathrm{~N}$ RMS accuracy. This was determined by applying $10 \mathrm{~Hz}$ bandlimited loads from $0-5 \mathrm{~N}$ and up to $30^{\circ}$ incidence angle. Calibration was performed in $37^{\circ} \mathrm{C}$ water to match in vivo thermal conditions.

\section{Force Control with Feed-Forward Motion Information}

Safety is a major concern when employing robotics in surgery. In particular, a damped, stable system is desired to ensure that the robot will not oscillate in response to sudden heart motions. Unfortunately, this is at odds with maintaining fast system response to reject force disturbances. It can be difficult to meet both criteria simultaneously when small, unknown time delays are present in the system. Feed-forward target motion information can improve force controller performance against a moving target [9] and in this section we explore its use to enhance safety and performance in the context of beating heart surgery.

The motion compensation instrument may be modeled as a mass $m$ and damper $b$ subjected to a commanded actuator force $f_{a}$ and environment contact force $f_{e}$. The damper $b$ captures the effects of friction in the instrument, friction at the insertion point to the heart, and fluid motion. Approximating the environment as a spring of stiffness $k_{e}$ yields the following system dynamics

$$
m \ddot{x}+b \dot{x}=f_{a}-k_{e}\left(x-x_{e}\right),
$$




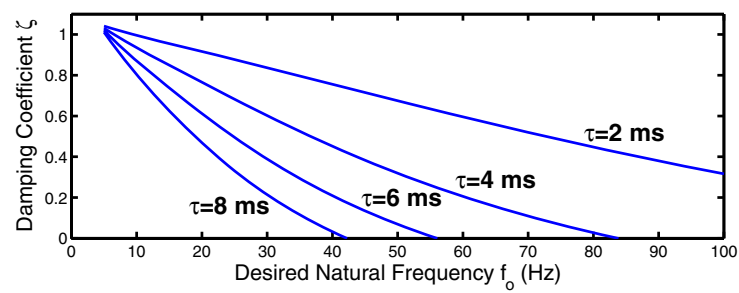

Fig. 4. Damping over desired natural frequency for small delays. $\zeta<0$ is unstable

where $x$ is the instrument tip position and $x_{e}$ would be the position of the tissue target if it were not deformed. Now consider a standard force error negative feedback control law [8]

$$
f_{a}=f_{d}+K_{f}\left(f_{d}-f_{e}\right)-K_{v} \dot{x},
$$

where $K_{f}$ and $K_{v}$ are controller gains and $f_{d}$ is the desired force. For brevity, we henceforth refer to the controller of (2) simply as force control. Combining (1) and (2) and applying the Laplace transform gives the contact force relationship

$$
F_{e}(s)=\frac{\frac{k_{e}}{m}\left(1+K_{f}\right)}{C(s)} F_{d}(s)-\frac{k_{e} s\left(s+\frac{K_{v}+b}{m}\right)}{C(s)} X_{e}(s)
$$

where $C(s)=s^{2}+\frac{K_{v}+b}{m} s+\frac{k_{e}}{m}\left(1+K_{f}\right)$ is the closed-loop characteristic equation. Eq. (3) makes explicit that target motion $x_{e}$ is a disturbance that perturbs $f_{e}$ from $f_{d}$. Controller gains $K_{f}$ and $K_{v}$ must be chosen both for system stability and good rejection of $x_{e}$. The latter can be achieved by setting the natural frequency of the system much greater than the motion bandwidth of $x_{e}$. This also makes the system vulnerable to high frequency noise.

Suppose we would like to set the damping coefficient $\zeta=1.05$ for a particular choice of the natural frequency $f_{o}$ using second order system design techniques [10]. Viscoelasticity in the force sensor can result in small time delays that severely reduce the actual $\zeta$ at large $f_{o}$ (Fig. 4). Noting that heart motion has significant spectral components up to at least $10 \mathrm{~Hz}$ [45]6], it is clear that it is difficult to obtain sufficient system bandwidth to safely reject $x_{e}$ without precise knowledge of the time delay $\tau$. Furthermore, empirically tuning the controller during a procedure is unfeasible for safety reasons.

An alternative strategy employs feed-forward motion information in the controller. Consider the control law

$$
f_{a}=f_{d}+K_{f}\left(f_{d}-f_{e}\right)+K_{v}\left(\hat{\dot{x_{e}}}-\dot{x}\right)+b \hat{\dot{x_{e}}}+m \hat{\dot{x}_{e}}
$$

which is (2) augmented with feed-forward estimates of the target velocity $\hat{\vec{x}}_{e}$ and acceleration $\hat{\dot{x}_{e}}$. The contact force equation from (3) becomes

$$
F_{e}(s)=\frac{\frac{k_{e}}{m}\left(1+K_{f}\right)}{C(s)} F_{d}(s)-\frac{k_{e} s\left(s+\frac{K_{v}+b}{m}\right)}{C(s)}\left(X_{e}(s)-\hat{X}_{e}(s)\right) .
$$


Observe that the use of feedforward terms $\hat{\dot{x_{e}}}$ and $\hat{\dot{x}_{e}}$ enable the cancellation of the disturbance $x_{e}$ without the need to greatly increase the natural frequency of the system. The controller can then be designed with low natural frequency to avoid the effects of delay on damping and stability.

\section{Tissue Motion Estimation with 3D Ultrasound}

To obtain the motion terms needed in the feed-forward controller, we must first determine the position of the tissue in $3 \mathrm{D}$ ultrasound. To do this, we employ the real-time tissue segmentation algorithm from 3, which uses the instrument to designate a tissue target in the 3D ultrasound volumes. Fig. 5 depicts using this method to track a point on the mitral valve annulus in a beating porcine heart.

As in previous work [6], we model heart motion as a time-varying Fourier series with an offset and truncated to $m$ harmonics

$$
x_{e}(t)=c(t)+\sum_{i=1}^{m} r_{i}(t) \sin \left(\theta_{i}(t)\right)
$$

where $c(t)$ is the offset, $r_{i}(t)$ are the harmonic amplitudes, and $\theta_{i}(t) \triangleq i \int_{0}^{t} \omega(\tau) d \tau$ $+\phi_{i}(t)$, with heart rate $\omega(t)$ and harmonic phases $\phi_{i}(t)$. Prior to contact, measurements from the tissue tracker are used to train an extended Kalman filter to provide estimates of the model parameters $\hat{c}(t), \hat{r}_{i}(t), \hat{\omega}(t)$, and $\hat{\theta}_{i}(t)$. These parameters are used to generate smooth feed-forward velocity and acceleration terms for the force controller of (4) using the derivative equations

$$
\hat{\dot{x}}_{e}(t)=\sum_{i=1}^{m} \hat{r}_{i}(t) i \hat{\omega}(t) \cos \left(\hat{\theta}_{i}(t)\right), \quad \hat{\dot{x}_{e}}(t)=-\sum_{i=1}^{m} \hat{r}_{i}(t)(i \hat{\omega}(t))^{2} \sin \left(\hat{\theta}_{i}(t)\right) .
$$

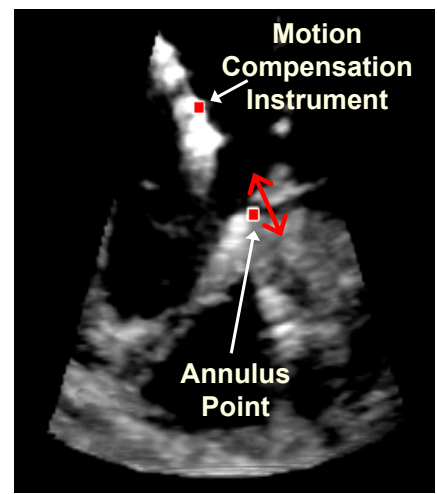

Fig. 5. Real-time 3D ultrasound tissue tracking. Squares denote instrument with force sensor and the mitral annulus.

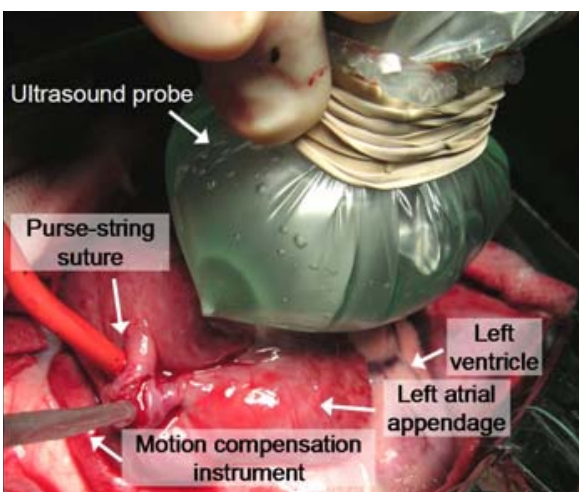

Fig. 6. In vivo experiment setup 
After contact, updates to the filter are stopped because the robot interacts with the tissue, causing subsequent position measurements to no longer be representative of the feed-forward (i.e. desired) tissue motion trajectory.

\section{In Vivo Validation}

\subsection{Experimental Setup}

In vivo validation was performed in a beating Yorkshire pig heart model (Fig.6). The tip of the motion compensation instrument was inserted into the left atrial appendage and secured by a purse-string suture. The 3D ultrasound probe (SONOS 7500, Philips Medical) was positioned epicardially on the left side of the heart to image the mitral valve and instrument. The surgeon was instructed to hold the instrument tip against the mitral annulus with a constant $2.5 \mathrm{~N}$ force for approximately $30 \mathrm{~s}$ under three conditions: manual (i.e. rigid instrument with no robot control), force control, and feed-forward force control. Controller gains were designed for $\zeta=1.05, f_{o}=8 \mathrm{~Hz}$ based on system identification of the parameters $m=0.27 \mathrm{~kg}, b=18.0 \mathrm{Ns} / \mathrm{m}$, and preliminary estimates of mitral annulus stiffness $k_{e}=133.0 \mathrm{~N} / \mathrm{m}$. Contact forces were visually displayed to the surgeon during the task and recorded for offline assessment. Three trials were attempted for each condition. The experimental protocol was approved by the Children's Hospital Boston Institutional Animal Care and Use Committee.
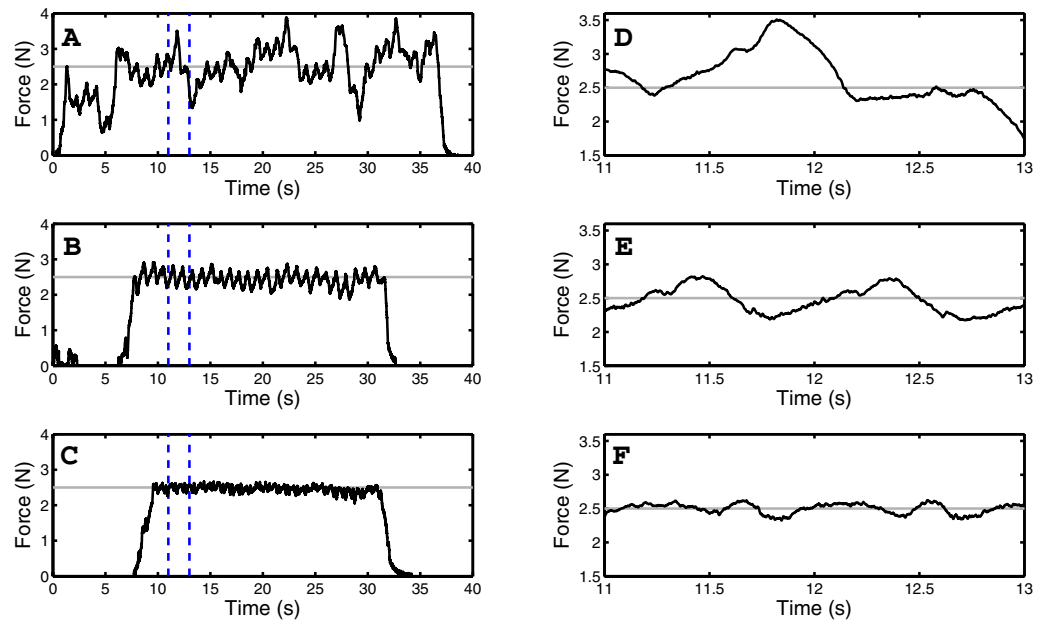

Fig. 7. Example contact forces for (A) manual, (B) force control, and (C) feed-forward force control. Corresponding enlarged views of the dashed regions in $(\mathrm{A}, \mathrm{B}, \mathrm{C})$ are shown in (D, E, F). The desired contact force of $2.5 \mathrm{~N}$ is indicated (horizontal line). Data was drawn from the trials with the lowest standard deviations. 


\subsection{Results}

Fig. 7 provides example force traces for the task executed manually, with force control, and with feed-forward force control. Averaged across all trials, manual contact with the annulus yielded force standard deviations of $0.48 \pm 0.06 \mathrm{~N}$ (mean \pm std error). Force control reduced these deviations to $0.22 \pm 0.01 \mathrm{~N}$ with clear statistical significance in a two-sided t-test $(p=0.012)$. Feed-forward force control reduced the deviations to approximately $25 \%$ of the manual case $(0.11 \pm$ $0.02 \mathrm{~N}, p=0.017)$. Statistical significance was also found between the force control and feed-forward control conditions $(p=0.009)$. These results are summarized in Fig. 8A. The third trial for the feed-forward force controller is omitted because the animal showed reduced viability at the end of the experiment. Performance was nearly equal to the standard force controller in this trial.

Force and feed-forward force control also reduced peak-to-peak forces (Fig. 8B). Manual use of the instrument gave swings in the contact force of $2.57 \pm 0.29 \mathrm{~N}$. Force control and feed-forward force control reduced these values to $1.16 \pm 0.10 \mathrm{~N}$ and $0.65 \pm 0.04 \mathrm{~N}$, respectively. Statistical significance was found between all conditions at $p<0.05$.
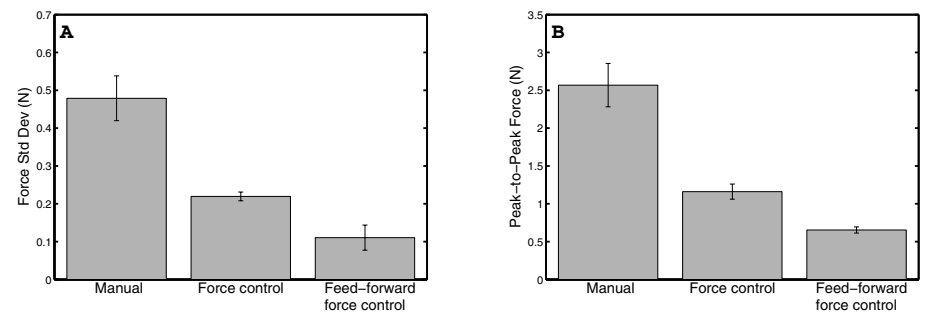

Fig. 8. Disturbance rejection measured by (A) standard deviation of forces and (B) peak-to-peak forces. Mean \pm std error is shown

\section{Discussion and Conclusions}

In vivo results indicate that safe, accurate robotic force stabilization is feasible inside of the beating heart. The system introduced here successfully exploits near periodicity in heart motion to generate smooth estimates of tissue velocity and acceleration from noisy 3D ultrasound imaging for a feed-forward force controller. As discussed before, the use of such a controller enables conservative tuning for increased stability and damping while allowing high bandwidth force disturbance rejection. To the authors' knowledge, this is the first in vivo demonstration of force control in the beating heart.

Without robotic stabilization, placement of the instrument against the mitral annulus gave peak-to-peak force swings of $2.57 \mathrm{~N}$, which is large compared to the desired $2.5 \mathrm{~N}$ force set point. Standard force error feedback control reduced this fluctuation by $50 \%$ and the full feed-forward controller using $3 \mathrm{D}$ 
ultrasound motion information reduced it by another $50 \%$. In the case of the feed-forward controller, the standard deviation of the contact forces was stabilized to $0.11 \mathrm{~N}$.

In all of the force control experiments, the surgeon expressed greater confidence in instrument manipulation against the beating mitral annulus, with the feed-forward controller subjectively better than the standard force controller. These findings suggest that robotic force control may be an effective aid to the surgeon for beating heart mitral annuloplasty. We note, however, that a potential limitation of the current study is that manual tasks were done with a (nonactuated) motion compensation instrument, which is heavier than typical surgical tools. Also, an analysis of the feed-forward controller performance in conditions of noisy force measurements and motion estimates is left for future work.

While this study focused on beating mitral valve annuloplasty, we envision that the fusion of force and position sensing in a feed-forward force controller will be amenable to other beating heart procedures where there is significant and extended contact with tissue, such as catheter ablation treatment for atrial fibrillation. It may also be useful for ongoing research in robotically-assisted coronary artergy bypass graft [4], where small, delicate vessels are handled.

Acknowledgments. This work is supported by the US National Institutes of Health under grant NIH R01 HL073647-06.

\section{References}

1. Murkin, J., Boyd, W., Ganapathy, S., Adams, S., Peterson, R.: Beating heart surgery: why expect less central nervous system morbidity? Annals of Thoracic Surgery 68, 1498-1501 (1999)

2. Falk, V.: Manual control and tracking - a human factor analysis relevant for beating heart surgery. Annals of Thoracic Surgery 74, 624-628 (2002)

3. Yuen, S.G., Kesner, S.B., Vasilyev, N.V., del Nido, P.J., Howe, R.D.: 3D ultrasound-guided motion compensation system for beating heart mitral valve repair. In: Metaxas, D., Axel, L., Fichtinger, G., Székely, G. (eds.) MICCAI 2008, Part I. LNCS, vol. 5241, pp. 711-719. Springer, Heidelberg (2008)

4. Ginhoux, R., Gangloff, J., de Mathelin, M., Soler, L., Sanchez, M.M.A., Marescaux, J.: Active filtering of physiological motion in robotized surgery using predictive control. IEEE Transactions on Robotics 21(1), 27-79 (2006)

5. Bebek, O., Cavusoglu, M.: Intelligent control algorithms for robotic assisted beating heart surgery. IEEE Transactions on Robotics 23(3), 468-480 (2007)

6. Yuen, S., Kettler, D.T., Novotny, P.M., Plowes, R.D., Howe, R.D.: Robotic motion compensation for beating heart intracardiac surgery. International Journal of Robotics Research (in press, 2009)

7. Puangmali, P., Liu, H., Althoefer, K.: Optical fiber sensor for soft tissue investigation during minimally invasive surgery. In: Proc. IEEE ICRA (2008)

8. Siciliano, B., Villani, L.: Robot Force Control, 1st edn. Springer, Heidelberg (1999)

9. Schutter, J.D.: Improved force control laws for advanced tracking applications. In: Proc. IEEE ICRA (1988)

10. Ogata, K.: Modern Control Engineering, 4th edn. Prentice-Hall, Englewood Cliffs (2001) 